

J. bio-sci. 18: 27-33, 2010

ISSN 1023-8654

http://www.banglajol.info/index.php/JBS/index

\title{
RESCUE OF REFRACTORY SPLICING OF CFTR EXON 12 PATHOLOGICAL MUTATIONS G576A/G48C USING ANTISENSE OLIGONUCLEOTIDES
}

\author{
Ariful Haque* \\ International Centre for Genetic Engineering and Biotechnology (ICGEB), 34149 Trieste, Italy
}

\begin{abstract}
Context: Pathological mutation in the human CFTR (Cystic Fibrosis Transmembrane conductance Regulator) exon 12 sequences disrupts splicing enhancer that leads to cystic fibrosis disease.

Objectives: Human CFTR exon 12 architecturally consists of two exonic regulatory silencers (Exonic Splicing Silencer / ESS) and an enhancer (Exonic Splicing Enhancer / ESE). In this study it was aimed to block the silencer activity as well as to introduce well known enhancer sequence along with the RNA to rescue CFTR exon 12 inclusion for therapeutic purpose.

Materials and Methods: In order to alter the pathologic RNA splicing event of CFTR exon 12 two oligonucleotides (ESE-A and ESS-B) were designed that, although complementary to the target RNA sequence but do not interfere in RNA processing. The oligonucleotide ESE-A additionally contains a non-complementary tail sequence mimicking ESE sequence. In this way the ESE were designed consequently for blocking silencer activity and enhance splice site selection. Furthermore, these oligonucleotides were designed based on several well known modified nucleotide sequences to provide stability from in vivo degradation.

Results: Naturally occurring pathological point mutation G576A/G48C in CFTR exon 12 causes complete skipping of the exon from full transcript. This study shows that cotransfection of oligonucleotides ESS-B along with CFTR exon 12 minigene carrying G576A/G48C mutation can rescue exon skipping.

Conclusion: The use of oligonucleotide ESS-B to enhance expression of latent CFTR exon 12 may ultimately be of therapeutic use for cystic fibrosis patient carrying pathological mutation in CFTR exon 12.
\end{abstract}

Keywords: CFTR exon 12, RNA splicing, Antisense Oligonucleotides.

\section{Introduction}

Cystic fibrosis (CF), also known as mucoviscoidosis is an autosomal recessive genetic disease that mostly affects the entire body causing progressive disability and early fatality. It is caused due to the mutation of CFTR (Cystic Fibrosis Transmembrane conductance Regulator) gene. The frequency of the disease differs among ethnic groups. However, it is more common in the Caucasian with an incidence of 1 in every 3,300 whilst within Hispanics the occurrence is 1 in 9,500. Surprisingly, it is an even rarer disorder in native Africans and Asians, where it is estimated to occur in less than 1 in 50,000 individuals (Goss and Rosenfeld 2004).

Cystic fibrosis is an extremely heterogeneous disease both in terms of the age of onset and for the clinical features. A number of individuals escape detection in the first decade or two of life, often because symptoms are mild. However, the typical patient with CF generally shows symptoms like thick production of mucus, which causes an obstructive chronic lungs disease, exocrine pancreatic insufficiency, intestinal obstruction of ileum in the newborns (meconium ileus) and sterility in $95 \%$ of men and in 10\% of women (Kerem and Kerem 1996, Koch and Hoiby 1993).

The gene CFTR contains 27 exons and transcribes the mature mRNA of 6kb. CFTR is a membrane associated glycoprotein of $169 \mathrm{kDa}$ and consists of 1480 amino acid residues (Gregory et al. 1990). Structurally the protein has two nucleotide binding domains (NBD1 and NBD2), one regulatory domain (R) and two hydrophobic transmembrane domains (each consist of six membrane spanning segments) (Riordan et al. 1989). The NBD1 subdivision of the protein is encoded by exon 9 through exon 12. In particular, exon

* Present address: Institute of Biological Sciences, University of Rajshahi, Rajshshi-6205, Bangladesh; E-mail: haque@ru.ac.bd. 
12 encodes amino acid spanning 560 to 588 towards the $C$ terminal of the NBD1 (Lewis et al. 2005). In brief, the protein functions to regulate the channel through phosphorylation of the R and NBDs. The proposed mechanism is that partial phosphorylation of the R domain cause the dimerization with NBD1, which leads to the opening of the gate at the transmembrane domain. Whereas, complete phosphorylation of the $\mathrm{R}$ domain cause the closing of the gate by interacting with the NBD2 (Bompadre et al. 2005). Mutation to any of these functional domains produces defective proteins and leads to disease.

Over recent years, the pathological alterations that can be directly linked with aberrant splicing processes have grown exponentially, and the study of the complex network interactions between defective splicing and occurrence of disease has become a central issue in the medical research field (Faustino and Cooper 2003, Garcia-Blanco et al. 2004, Nissim-Rafinia and Kerem 2005). A general view of alternative splicing implies focusing on specific sequence elements, some interacting predominantly with SR (Serine Arginine rich) proteins that act in a way as to include the exon (splicing enhancers) and some with hnRNPs (Heterogenous ribonuclear protein) that promote exon skipping (splicing suppressors). In theory, this mechanism should be a simple task for the exonic mutation analysis. However, this strategy often produces ambiguous results, particularly in the situation where the cis-elements are extremely overlapped like in CFTR exon 12. Previously, we showed that human flanking sequence have ESS sequences in both the 5'ss and 3'ss flanking regions and CERES 2 (Composite Exonic Regulatory Element of Splicing) at the central sequence (Haque et al. 2010). Mutations like G576A/G48C and Y577Y/C52T in the CERES 2 sequence in fact inactivates it's enhancing ability, which is why both of the flanking silencer completely abolish exon selection and leads to cystic fibrosis (Haque et al. 2010, Pagani et al. 2003a).

Here in this study a possible therapeutic mechanism for rescuing CFTR exon 12 in the presence of G576A/G48C mutation has been described. In this regard, oligonucleotides were designed following available strategy of blocking ESS by complementary sequence and introducing ESE in a tail (Skordis et al. 2003). The result shows that only obstruction of ESS near the 3' end of CFTR exon 12 can moderately increase exon inclusion in a minigene system.

\section{Materials and Methods}

Hybrid minigene constructs: Human CFTR exon 12 minigene constructs G48C and WT (Wild Type) have been previously described (Pagani et al. 2003a, 2005). In brief, G48C mutation in the exon were introduced by PCR-directed mutagenesis using specific primers and cloned inside the Ndel restriction site of the PTB plasmid. Primer sequences for each described mutants can be provided up on request.

Oligonucleotides and RNA secondary structure prediction: ESE-A and ESS-B oligonucleotides were purchased from Biosysthesis, USA. The ESE-A RNA oligo sequence were 5'asgsgsasgsgacggaggacggaggacaGoUCoUAoAUoAAoAUoACOAAOAUOCAoGCo-3' and the ESS-B sequence were 5'-GoUCoUAoAUoAAoAUoACoAAoAUoCAoGCo-3'. Here all the nucleotides with capital letter represent complementary sequence of the targeted RNA sequences and nucleotides with "o" represents LNA (Locked Nucleic Acid). For in vivo stability all the RNA sequences were 2'-O-Methylated.

RNA secondary structure predictions were performed using the freely available mfold server. This web server uses mfold (version 3.2) by Zuker (2003). (http://mfold.bioinfo.rpi.edu/cgi-bin/rna-form1.cgi)

Cell culture, transfections, and reverse transcription-PCR (RT-PCR) analysis: HeLa cells were cultured in Dulbecco's modified Eagle's medium with Glutamax (Invitrogen) in standard conditions. The G48C minigene used for transfection were purified using phenol-choloroform extraction followed by a sephacryl S-400 (GE healthcare) column purification step. HeLa cells were plated at a concentration of $2.8 \times 10^{5}$ to achieve 80 to $90 \%$ confluence. At the expected confluence (usually on the next day), $500 \mathrm{ng}$ of plasmid DNA were mixed 
with ESE-A and ESS-B oligonucleotides separately and transfected the cells using Oligofectamine transfection reagents (Qiagen). Finally, after 24 hours total RNA was extracted using TRIreagent solution (Ambion). One microgram of total RNA was used in the retrotranscription reaction with random primers and Moloney murine leukemia virus enzyme (Invitrogen). Spliced products from the transfected minigene were obtained using primers Bra2 5'taggatccggtcaccaggaagttggttaaatca-3' and $\alpha$ 2-3 5' caacttcaagctcctaagccactgc- $3^{\prime}$. PCR conditions were the following: $94^{\circ} \mathrm{C}$ for $5 \mathrm{~min} ; 94^{\circ} \mathrm{C}$ for $30 \mathrm{~s}, 55^{\circ} \mathrm{C}$ for 30 $\mathrm{s}$, and $72^{\circ} \mathrm{C}$ for $30 \mathrm{~s}$ for 30 cycles; and $72^{\circ} \mathrm{C}$ for $7 \mathrm{~min}$ for the final extension. PCRs were optimized to be in the exponential phase of amplification and products were routinely fractionated in 1.8\% (w/v) agarose gels. Visible exon inclusion bands in gels were later quantified using Image J (Open source Java) software.

\section{Results}

Effect of the pathological missense mutation G576A/G48C on CFTR exon 12 in the CERES 2 element: In normal conditions, the level of CFTR exon 12 skipping from the full mRNA transcript is variable and ranges from 5\%-30\% (Hull et al. 1994). Loss of this exon removes the highly conserved region from the NBD1 and produces non-functional CFTR protein. Earlier studies showed that miss-sense mutations G576A/G48C in the CFTR exon 12 causes cystic fibrosis by affecting the splicing of the exon rather than only changing the amino acid (Pagani et al. 2003a). Interestingly, these pathological mutations were initially mentioned as polymorphism in CFTR mutation. In order to understand the role of the mutation in splicing, Pagani et al (2003a) at first analyzed the RNA from patient's nasal epithelial tissue. Subsequently, they carried the analysis into a minigene system to better understand the effect on splicing regulation. In the minigene system, along with exon 12 they inserted 333nt from intron 11 and 270nt from intron 12 to obtain a reliable splicing pattern that mimicked the endogenous splicing pattern. Transfection of G576A/G48C minigene along with WT human CFTR exon 12 in HeLa cell line yielded similar results, that is WT minigene showed $\sim 80 \%$ exon inclusion, where as G576A/G48C showed complete exon skipping (Fig 1).

A

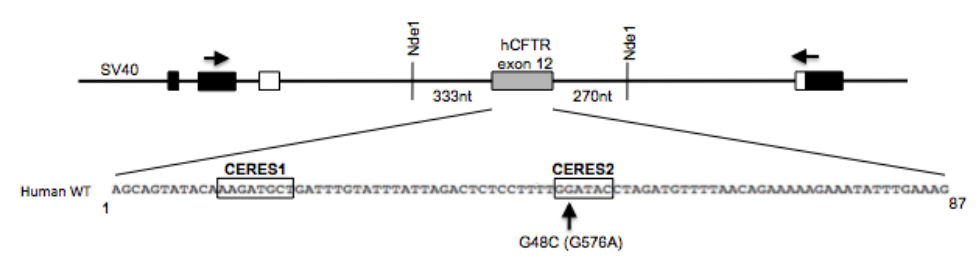

B

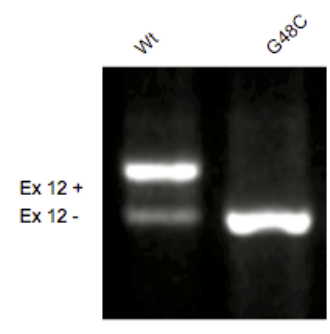

Fig. 1: (A). Schematic presentation of the hybrid minigene used in transfection experiments. The $\alpha$-globin, fibronectin EDB, and human CFTR Exon 12 are shown as black, white and gray boxes, respectively. The sequence of CFTR exon 12 and position of the CERES1 and CERES2 elements are reported in full. Mutation G576A/G48C is indicated with arrow. (B) The amplified RTPCR products stained with ethidium bromide are shown in the bottom panels. Spliced transcripts are shown with Ex.12+ for inclusion and Ex.12- for exclusion of the exon. 
A

Esea : 5' aggaggacggaggacggaggacamgagucuanuanauacanauc 3'

ESS B : 5' agagucuanuaAauacaanuc $3^{\prime}$

B

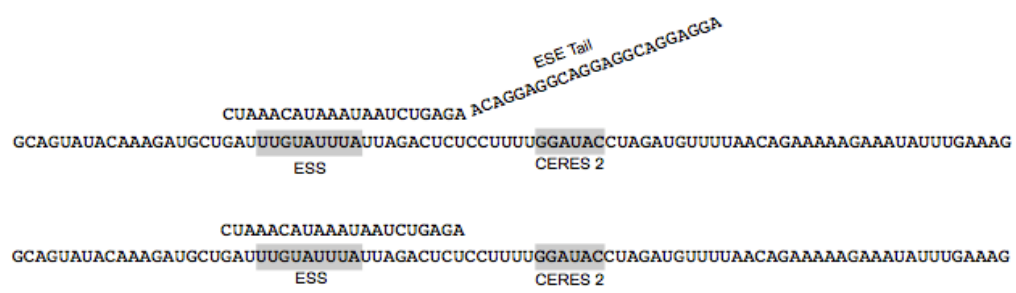

Fig. 2: (A) Sequence of the antisense RNA that are used in order to rescue G576A/G48C mutation. (B) Schematic presentation of the targeted sequence of CFTR exon 12. Gary boxes shows the ESS and CERES 2 sequence of the exon. Projected sequence of the oligonucleotide shows the non complementary part of the oligo carrying ESE.

Blocking of ESS sequence spanning from nt 19-39 increases exon inclusion: Two antisense oligonucleotides ESE-A and ESS-B were designed that were targeting same sequence of CFTR exon 12 spanning from 19-39 (Fig 2). However, ESE-A was carrying a non-complementary well known enhancer sequence that interacts SF2/ASF protein and capable of enhancing splicing (Liu et al. 1998, Skordis et al. 2003). However, it is now widely accepted that the local structure of the pre-mRNA can affect cis-acting elements accessibility to transacting factors. In particular, secondary structure can act by hiding or masking enhancers, and silencers, with the obvious relative effects (Buratti and Baralle 2004). For this reason, in order to check whether the oligonucleotides are accessible to target site it was predicted in silico based RNA secondary structure. The structure analysis shows that the target RNA sequence is predominantly open in a bulge in high free energy (Fig 3). It was presumed that this flexible structure is well enough for oligonucleotide accessibility in HeLa cell physiological condition. However, the principal limitation in identifying the role of secondary structure is that our predictive abilities are still rather limited and safe judgment should be made only through implementation of additional functional studies and experimental probing.

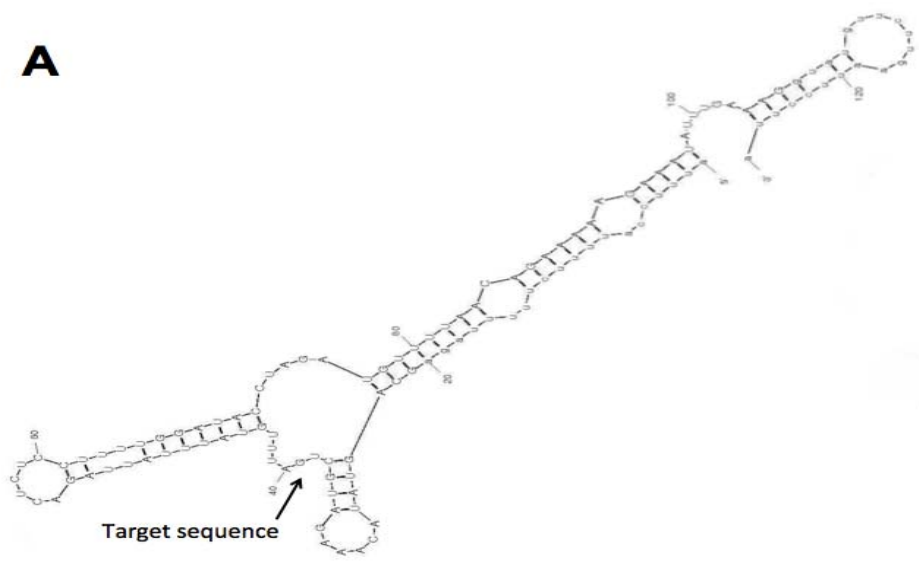

Fig. 3: (A) In silico prediction of CFTR exon 12 secondary structure flanked by $20 \mathrm{nt}$ of intronic sequences of both splice sites. Arrowed bulge in the structure shows the binding site of the oligonucleotide 
In order to verify the effect of the oligonucleotide, HeLa cells were cotransfected at various concentrations oligonucleotides between 200 to $500 \mathrm{mM}$. At the presence of Y576A/G48C mutation, CFTR exon 12 completely skips from mature mRNA but cotransfection with ESS-B oligonucleotide shows nearly $5 \%$ increase of exon inclusion even at $200 \mathrm{mM}$ concentration. Increased concentration to $500 \mathrm{mM}$ of oligonucleotide ESS-B showed up to $\sim 20 \%$ exon inclusion (Fig 4). This is probably because of the ESS-B oligo was blocking the interaction of trans acting factors that was responsible for exerting the effect of ESS in exon skipping. Although we expected to see the ESE-A oligo work efficiently because it carries an enhancer sequence but didn't work, possibly due to the fact that the ESE-A oligonucleotide was too long to access to the target site or may be it was constraining the general RNA splicing ability.

A

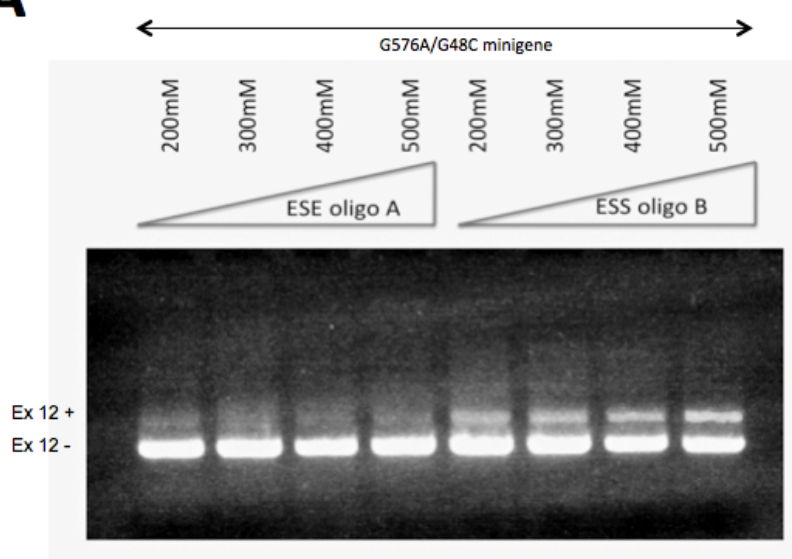

B

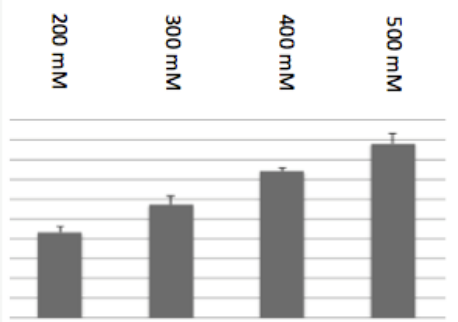

Fig. 4: (A) Effects of cotransfecting the increasing concentration of ESE-A and ESS-B oligonucleotides along with G576A/G48C minigene in Hela cells. RT-PCR samples are stained with ethidium bromide and run on a 1.8\% agarose gel. Exon inclusion and skipping are is shown by Ex12+ and Ex12-, respectively. (B) Quantification of rescued exons by ESS-B Oligonucleotides

\section{Discussion}

The number of mutations in the CFTR gene that are causing the disease symptoms is growing every day. The complete list of mutation identified up to now can be found at the world web access http://www.genet.sickkids.on.ca. In most of the cases, the pathological effect of single base substitutions in CFTR gene were considered to be based on the change in coding sequence (or were mis-classified as benign polymorphism databases). Nowadays, several examples have been shown that many of these changes affect splicing pattern of the gene instead changing the specific amino acids (Pagani et al. 2003ab, Zielenski et al. 1995). However, the symptoms among CF patients differ a lot and it is hard to correlate the phenotypic symptoms with the genotype. Abnormal electrolyte composition of sweat is common for virtually all patients with classical CF. However, severity of the disease is strongly affected by environmental and secondary genetic factors (CF modifiers) (Zielenski and Tsui 1995, Zielenski 2000). Interestingly, human cells require only $12 \%$ of the full length CFTR mRNA for functional CAMP mediated epithelial conductance. Mutation like G576A/G48C and Y577Y/C52T causes complete skipping of exon 12, which encodes the part of important NBD1 domain of the protein. The present results shows that blocking of the silencer near the 3 'ss by ESS-B oligo is capable of rescuing $20 \%$ of the full length mRNA. However, the experiment was carried out in a minigene context, which contains a strong beta globin promoter with viral SV40 enhancer. Presence of SV40 enhancer produces large number of RNA compared to transfected oligonucleotides. 
Notable difference between minigene transcribed RNA and transfected oligonucleotide ratio possibly reduced the percentage of full length RNA rescue. Therefore, further investigation of ESS-B oligo in a transcriptional landscape that is the patient cells or in vivo model, will be worthy for inventing successful gene therapy.

Antisense RNA mediated gene therapy may be the ultimate use for exon rescue. Several of the phosphorothioate (S-nucleotide) modified antisense RNA is in the clinical trial for viral infection and cancer treatment (Galderisi et al. 1999), however it should be considered in this approach that excess concentration of sulphur is toxic to cell. Another approach of trans splicing mediated CFTR RNA correction has been successful (Song et al. 2009) but for technical limitations trans splicing strategy remained successful for only short mRNA or for terminal exons of long mRNA. Thus, this method is hard to adapt for a central exon like CFTR 12. Approximately $30 \%$ of the synonymous mutation in CFTR exon 12 affects splicing (Pagani et al. 2005). In this regard the present approach of oligonucleotide mediated rescue of the exons has been previously successful for SMA (Spinal Muscular Atrophy) (Skordis et al. 2003) and now also can be a key solution not only for CFTR exon 12 but also for other central exon of long mRNAs.

\section{References}

Bompadre SG, Ai T, Cho JH, Wang X, Sohma Y, Li M, Hwang TC. 2005. CFTR gating I: Characterization of the ATP-dependent gating of a phosphorylation-independent CFTR channel (DeltaR-CFTR). J Gen Physiol 125, 361-375. http://dx.doi.org/10.1085/jgp.200409227 PMid:15767295 PMCid:1382195

Buratti E, Baralle FE. 2004. Influence of RNA Secondary Structure on the Pre-mRNA Splicing Process. Mol Cell Biol 24, 10505-10514. http://dx.doi.org/10.1128/MCB.24.24.10505-10514.2004 PMid:15572659 PMCid:533984

Faustino NA, Cooper TA. 2003. Pre-mRNA splicing and human disease. Genes Dev 17, 419-437. http://dx.doi.org/10.1101/gad.1048803

Galderisi U, Cascino A, Giordano, A. 1999. Antisense oligonucleotides as therapeutic agents. J Cell Physiol 181, 251-257. http://dx.doi.org/10.1002/(SICI)1097-4652(199911)181:2<251::AID-JCP7>3.0.CO;2-D

Garcia-Blanco MA, Baraniak AP, Lasda EL. 2004. Alternative splicing in disease and therapy. Nat Biotechnol 22, 535-546. http://dx.doi.org/10.1038/nbt964 PMid:15122293

Goss CH, Rosenfeld M. 2004. Update on cystic fibrosis epidemiology. Curr Opin Pulm Med 10, 510-514. http://dx.doi.org/10.1097/01.mcp.0000138994.46519.72 PMid:15510059

Gregory RJ, Cheng SH, Rich DP, Marshall J, Paul S, Hehir K, Ostedgaard L, Klinger KW, Welsh MJ, Smith AE. 1990. Expression and characterization of the cystic fibrosis transmembrane conductance regulator. Nature 347, 382-386. http://dx.doi.org/10.1038/347382a0 PMid:1699127

Haque A, Buratti E, Baralle FE. 2010. Functional properties and evolutionary splicing constraints on a composite exonic regulatory element of splicing in CFTR exon 12. Nucleic Acids Res 38, 647-659. http://dx.doi.org/10.1093/nar/gkp1040 PMid:19910374 PMCid:2811005

Hull J, Shackleton S, Harris A. 1994. Analysis of mutations and alternative splicing patterns in the CFTR gene using mRNA derived from nasal epithelial cells. Hum Mol Genet 3, 1141-1146. http://dx.doi.org/10.1093/hmg/3.7.1141 PMid:7526925

Kerem E, Kerem B. 1996. Genotype-phenotype correlations in cystic fibrosis. Pediatr Pulmonol 22, 387-395. http://dx.doi.org/10.1002/(SICI)1099-0496(199612)22:6<387::AID-PPUL7>3.0.CO;2-G http://dx.doi.org/10.1002/(SICI)10990496(199612)22:6<387::AID-PPUL7> 3.3.CO;2-Y

Koch C, Hoiby N. 1993. Pathogenesis of cystic fibrosis. Lancet 341, 1065-1069. http://dx.doi.org/10.1016/0140-6736(93)92422-P

Lewis HA, Zhao X, Wang C, Sauder JM, Rooney I, Noland BW, Lorimer D, Kearins MC, Conners K, Condon B. 2005. Impact of the deltaF508 mutation in first nucleotide-binding domain of human cystic fibrosis transmembrane conductance regulator on domain folding and structure. J Biol Chem 280, 1346-1353. http://dx.doi.org/10.1074/jbc.M410968200 PMid:15528182

Liu HX, Zhang M, Krainer AR. 1998. Identification of functional exonic splicing enhancer motifs recognized by individual SR proteins. Genes Dev 12, 1998-2012. http://dx.doi.org/10.1101/gad.12.13.1998

Nissim-Rafinia M, Kerem B. 2005. The splicing machinery is a genetic modifier of disease severity. Trends Genet 21, 480-483. http://dx.doi.org/10.1016/j.tig.2005.07.005 PMid:16039004

Pagani F, Stuani C, Tzetis M, Kanavakis E, Efthymiadou A, Doudounakis S, Casals T, Baralle FE. 2003a. New type of disease causing mutations: the example of the composite exonic regulatory elements of splicing in CFTR exon 12. Hum Mol Genet 12, 1111-1120. http://dx.doi.org/10.1093/hmg/ddg131 PMid:12719375

Pagani F, Buratti E, Stuani C, Baralle FE (2003b). Missense, nonsense, and neutral mutations define juxtaposed regulatory elements of splicing in cystic fibrosis transmembrane regulator exon 9. J Biol Chem 278, 26580-26588. http://dx.doi.org/10.1074/jbc.M212813200 PMid:12732620 
Pagani F, Raponi M, Baralle FE. 2005. Synonymous mutations in CFTR exon 12 affect splicing and are not neutral in evolution. Proc Natl Acad Sci USA 102, 6368-6372. http://dx.doi.org/10.1073/pnas.0502288102 PMid:15840711 PMCid:1088389

Riordan JR, Rommens JM, Kerem B, Alon N, Rozmahel R, Grzelczak Z, Zielenski J, Lok S, Plavsic N, Chou JL. 1989. Identification of the cystic fibrosis gene: cloning and characterization of complementary DNA. Science 245, 1066-1073. http://dx.doi.org/10.1126/science.2475911 PMid:2475911

Skordis LA, Dunckley MG, Yue B, Eperon IC, Muntoni F. 2003. Bifunctional antisense oligonucleotides provide a trans-acting splicing enhancer that stimulates SMN2 gene expression in patient fibroblasts. Proc Natl Acad Sci USA 100, 4114-4119. http://dx.doi.org/10.1073/pnas.0633863100 PMid:12642665 PMCid:153057

Song Y, Lou HH, Boyer JL, Limberis MP, Vandenberghe LH, Hackett NR, Leopold PL, Wilson JM, Crystal RG. 2009. Functional cystic fibrosis transmembrane conductance regulator expression in cystic fibrosis airway epithelial cells by AAV6.2-mediated segmental trans-splicing. Hum Gene Ther 20, 267-281. http://dx.doi.org/10.1089/hum.2008.173 PMid:19257851 PMCid:2855253

Zielenski J. 2000. Genotype and phenotype in cystic fibrosis. Respiration 67, 117-133. http://dx.doi.org/10.1159/000029497 PMid:10773783

Zielenski J, Tsui LC. 1995. Cystic fibrosis: genotypic and phenotypic variations. Annu Rev Genet 29, 777-807. http://dx.doi.org/10.1146/annurev.ge.29.120195.004021 PMid:8825494

Zielenski J, Markiewicz D, Lin SP, Huang FY, Yang-Feng TL, Tsui LC. 1995. Skipping of exon 12 as a consequence of a point mutation $(1898+5 G-->T)$ in the cystic fibrosis transmembrane conductance regulator gene found in a consanguineous Chinese family. Clin Genet 47, 125-132. http://dx.doi.org/10.1111/j.1399-0004.1995.tb03944.x

Zuker M. 2003. Mfold web server for nucleic acid folding and hybridization prediction. Nucleic Acids Res 31, 3406-3415 http://dx.doi.org/10.1093/nar/gkg595 PMid:12824337 PMCid:169194 